\section{(C) OPEN ACCESS}

\title{
Mechanical stress potentiates the differentiation of periodontal ligament stem cells into keratocytes
}

\author{
Jialin Chen, ${ }^{1}$ Wei Zhang, ${ }^{1}$ Ludvig J Backman, ${ }^{1}$ Peyman Kelk, ${ }^{1}$ Patrik Danielson ${ }^{1,2}$
}

- Additional material is published online only. To view please visit the journal online (http://dx.doi.org/10.1136/ bjophthalmol-2017-311150).

'Department of Integrative Medical Biology, Umeå University, Umeå, Sweden ${ }^{2}$ Unit of Ophthalmology, Department of Clinical Sciences, Umeå University, Umeå, Sweden

\section{Correspondence to}

Professor Patrik Danielson, Department of Integrative Medical Biology, Anatomy, Umeå University, Umeå, SE-901 87, Sweden; patrik.danielson@ umu.se

$J C$ and WZ contributed equally.

Received 8 August 2017 Revised 24 November 2017 Accepted 9 December 2017 Published Online First 6 January 2018
Check for updates

To cite: Chen J, Zhang W,

Backman LJ, et al.

Br J Ophthalmol

2018:102:562-569.

\section{ABSTRACT}

Aims To explore the role of corneal-shaped static mechanical strain on the differentiation of human periodontal ligament stem cells (PDLSCS) into keratocytes and the possible synergistic effects of mechanics and inducing medium.

Methods PDLSCs were exposed to 3\% static domeshaped mechanical strain in a Flexcell Tension System for 3 days and 7 days. Keratocyte phenotype was determined by gene expression of keratocyte markers. Keratocyte differentiation (inducing) medium was introduced in the Flexcell system, either continuously or intermittently combined with mechanical stimulation. The synergistic effects of mechanics and inducing medium on keratocyte differentiation was evaluated by gene and protein expression of keratocyte markers. Finally, a multilamellar cell sheet was assembled by seeding PDLSCs on a collagen membrane and inducing keratocyte differentiation. The transparency of the cell sheet was assessed, and typical markers of native human corneal stroma were evaluated by immunofluorescence staining.

Results Dome-shaped mechanical stimulation promoted PDLSCs to differentiate into keratocytes, as shown by the upregulation of $A L D H 3 A 1, C D 34, L U M, C O L /$ and COL $V$. The expression of integrins were also upregulated after mechanical stimulation, including integrin alpha 1, alpha 2, beta 1 and non-muscle myosin II B. A synergistic effect of mechanics and inducing medium was found on keratocyte differentiation. The cell sheets were assembled under the treatment of mechanics and inducing medium simultaneously. The cell sheets were transparent, multilamellar and expressed typical markers of corneal stroma.

Conclusion Dome-shaped mechanical stimulation promotes differentiation of PDLSCs into keratocytes and has synergistic effects with inducing medium. Multilamellar cell sheets that resemble native human corneal stroma show potential for future clinical applications.

\section{INTRODUCTION}

The light refraction function of the cornea is highly dependent on the well-organised collagen structures of the corneal stroma, with collagen type I (COL I) and type $\mathrm{V}(\mathrm{COL} \mathrm{V})$ as the main collagens in human. Keratocytes are cells residing in the corneal stroma. They express aldehyde dehydrogenase 3A1 (ALDH3A1), CD34 and two major proteoglycans: lumican (LUM) and keratocan (KERA). ${ }^{1}$ Primary keratocytes have been isolated and cultured in vitro to study the cell behaviours and function for many years. ${ }^{2}$ However, disadvantages of in vitro culturing primary keratocytes, such as decreased expression of keratocyte markers, ${ }^{1}$ low proliferation rate ${ }^{3}$ and insufficient extracellular matrix (ECM) deposition, ${ }^{4}$ hamper their application. Efficiently differentiating stem cells towards keratocyte lineage is a potential approach to continually obtain keratocytes with normal phenotype. Various stem cells derived from different tissues, such as adipose tissue, ${ }^{5}$ and corneal stroma itself ${ }^{6}$ have been used for keratocyte differentiation. Periodontal ligament stem cells (PDLSCs) is another promising alternative, as they are similar to keratocytes in developmental origin and proteoglycan expression. ${ }^{7}$ However, no attempts have been reported yet.

Keratocyte inducing medium (IM) has been developed for some years to differentiate stem cells into keratocytes. Different biological factors have been used in these attempts, including basic fibroblast growth factor-2 (FGF-2, also known as bFGF) ${ }^{56}$ and transforming growth factor-beta3 (TGF- $\beta 3){ }^{6} \mathrm{Wu}$ and collaborators compared the differentiation effect between FGF- 2 and TGF- $\beta 3$, alone and in combination, for up to 9 weeks. ${ }^{6}$ The best effect was achieved in the combination group, with higher expression of keratocytes markers and deposition of orthogonally oriented collagens. ${ }^{6}$ However, there is still a long way to go to achieve an ideal keratocyte differentiation protocol that is more convenient, effective and less time consuming.

The effect of mechanical stimulation has been reported in stem cell differentiation into, for instance, tenocytes $^{8}$ and chondrocytes. ${ }^{9}$ The cornea is a pressurised structure, with a dome-shaped strain on the cells inside. Our previous study was the first to show that 3\% dome-shaped static mechanical stimulation increases the expression of LUM, KERA, COL I and $\mathrm{COL} \mathrm{V}$ in primary human keratocytes. ${ }^{10}$ However, to our knowledge, there is no report on the effect of mechanical stimulation in stem cell differentiation towards keratocytes, nor on the possible synergistic effects of mechanics and chemical differentiation medium.

The current study aimed to evaluate the differentiation effect of mechanical strain on stem cells towards keratocytes and to explore the possible synergistic effects of mechanical strain (physical factor) and induction medium (biochemical factor). Finally, cell culturing with the optimal differentiation protocol was performed to form a cell sheet to study the potential significance in clinical applications.

\section{MATERIALS AND METHODS \\ Cell culture of human PDLSCs}

The study protocol was in accordance with the principles of the Declaration of Helsinki. Written informed consent was obtained from all patients. 


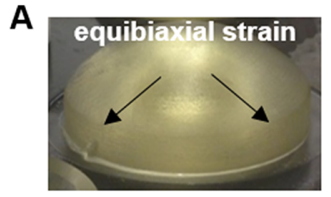

dome-shaped loading post

SM: static mechanics
B

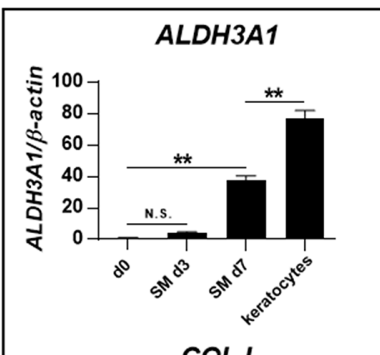

COLI

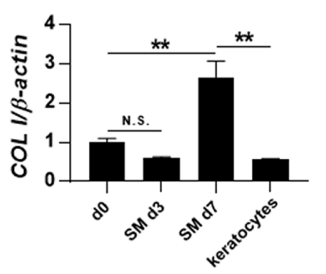

SCX

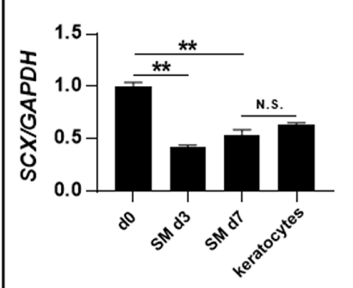

ITGA2

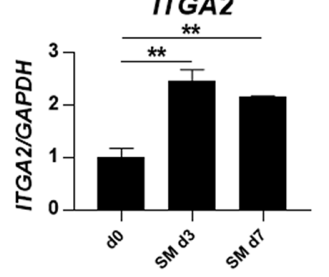

CD34

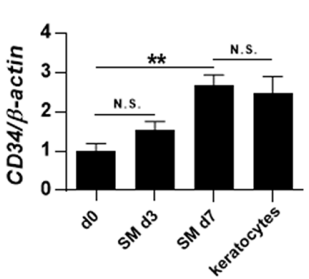

$\operatorname{COLV}$

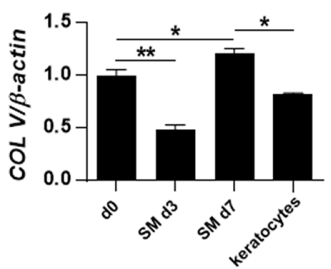

TNMD

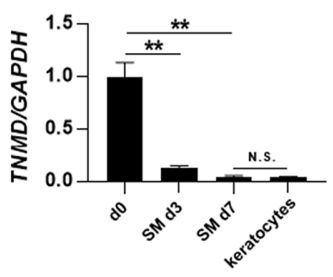

ITGB1

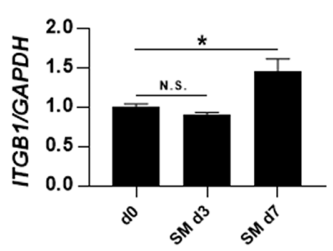

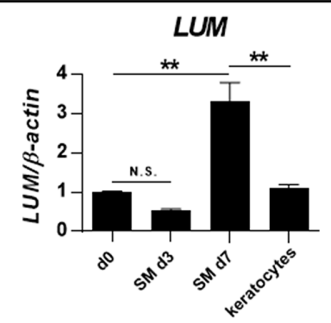

ACTA2

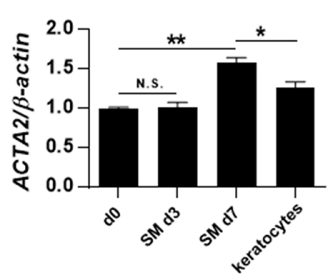

ITGA1

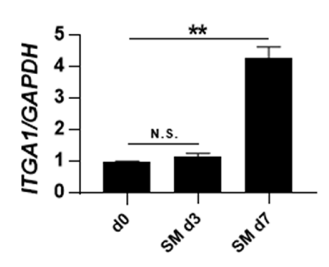

MYH10

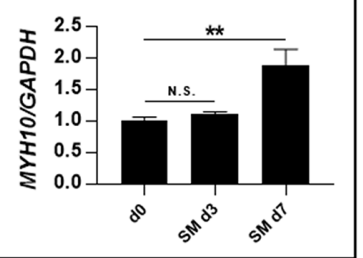

Figure 1 Dome-shaped mechanical stimulation promotes keratocyte differentiation. (A) A dome-shaped loading post was used to apply 3\% equibiaxial static strain on PDLSCs in the Flexcell Tension System for 3 days and 7 days. (B) Gene expression was evaluated by quantitative PCR, including the genes for keratocyte makers (ALDH3A1,CD34 and LUM), the main collagens of the corneal stroma (COL I and COL V), myofibroblast maker (ACTA2), tenocyte markers (SCX and TNMD) and integrins (ITGA1, ITGA2, ITGB1 and MYH10). Levels at day 0 (d0, unstimulated PDLSCs) were set as 1 . The expression was compared between $\mathrm{d} 0$ and day 3 after stimulation of static mechanics (SM d3), d0 and day 7 after stimulation of static mechanics (SM d7), d7 and primary in vitro cultured keratocytes at passage 2. " Significant difference at $P<0.05$. ${ }^{* *}$ Significant difference at $P<0.001$. N.S., no significant difference $(P \geq 0.05)$. PDLSCs, periodontal ligament stem cells.

Human PDLSCs from two patients were used in this study, which were isolated from surgically removed impacted third molars. The detailed isolation protocol and identification of PDLSCs could be found in online supplementary materials and methods. Human PDLSCs were cultured in growing medium, Minimum Essential Medium- $\alpha$ (Life Technologies, Grand Island, New York, USA) with 10\% fetal bovine serum (FBS, Life Technologies)) and 1\% penicillin-streptomycin (Life Technologies). Cells between passages three and six were used for the experiments. The PDLSCs used in the present project have recently been characterised in another study of ours. ${ }^{11}$

\section{Mechanical stimulation alone}

PDLSCs were seeded on Collagen I bonded Bioflex 6-well plate (Flexcell International Corporation, Burlington, North Carolina, USA). The plate was placed on a dome-shaped loading post of $25 \mathrm{~mm}$ diameter. The cells received $3 \%$ equibiaxial static mechanics (SM) in the Flexcell Tension System by stretching the collagen membranes of the plate around the dome-shaped loading post. ${ }^{10}$ Four millilitre media was used for each well of Bioflex 6-well plate and was changed on the second or third day. Samples were collected at day 0 (d0; unstimulated PDLSCs), day 3 (d3) and day 7 (d7) for quantitative PCR (qPCR) analysis. To evaluate the keratocyte differentiation efficacy on PDLSCs, cultured primary human limbal keratocytes (at passage 2) ${ }^{12}$ were used as positive controls.

\section{Combination of mechanical stimulation and IM}

The IM for keratocyte differentiation was prepared as described previously, ${ }^{6}$ concisely advanced Dulbecco's modified Eagle's medium (DMEM) (Life Technologies), $1 \mathrm{mM} \mathrm{L}$-ascorbic acid 2-phosphate (Sigma-Aldrich, St. Louis, Missouri, USA), $10 \mathrm{ng} /$ $\mathrm{mL}$ of the basic FGF-2 (Invitrogen, Carlsbad, California, USA) and $0.1 \mathrm{ng} / \mathrm{mL}$ of the TGF- $\beta 3$ (Sigma-Aldrich).

PDLSCs $\left(2 \times 10^{5} /\right.$ well) were seeded on Bioflex 6 -well plate and cultured in regular growing medium for 1 day before treatments. Samples were collected after 6 days of treatment for qPCR and Western blot. Samples at $\mathrm{d} 0$ (unstimulated PDLSCs) were 
A

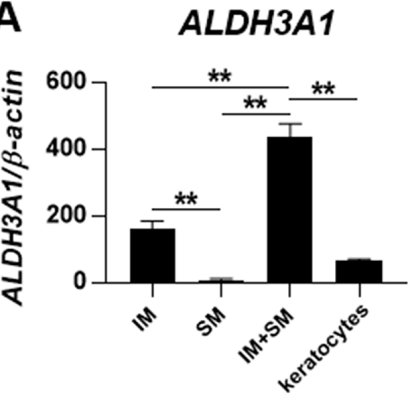

COL I

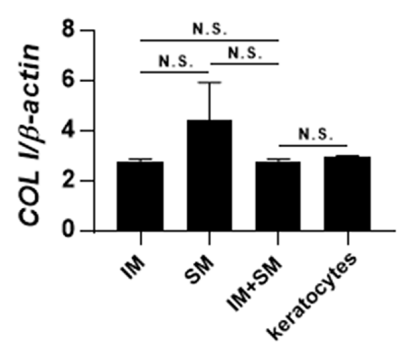

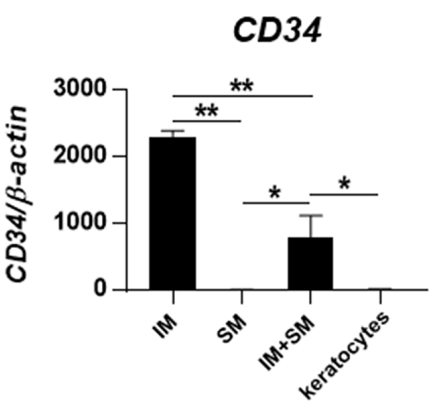

COL V

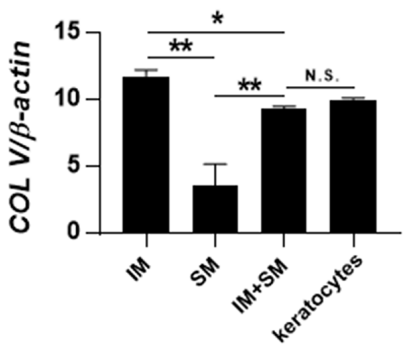

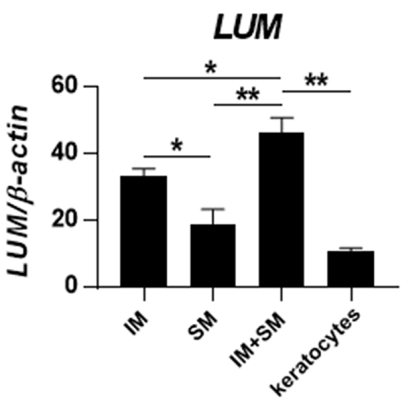

ACTA2

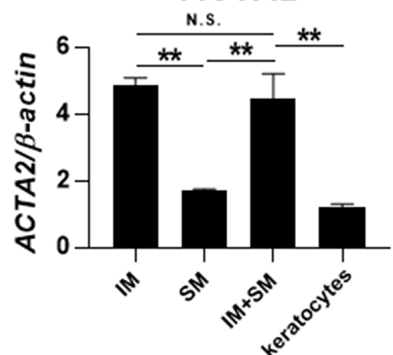

IM: inducing medium SM: static mechanics

PDLSC d0 was set as 1

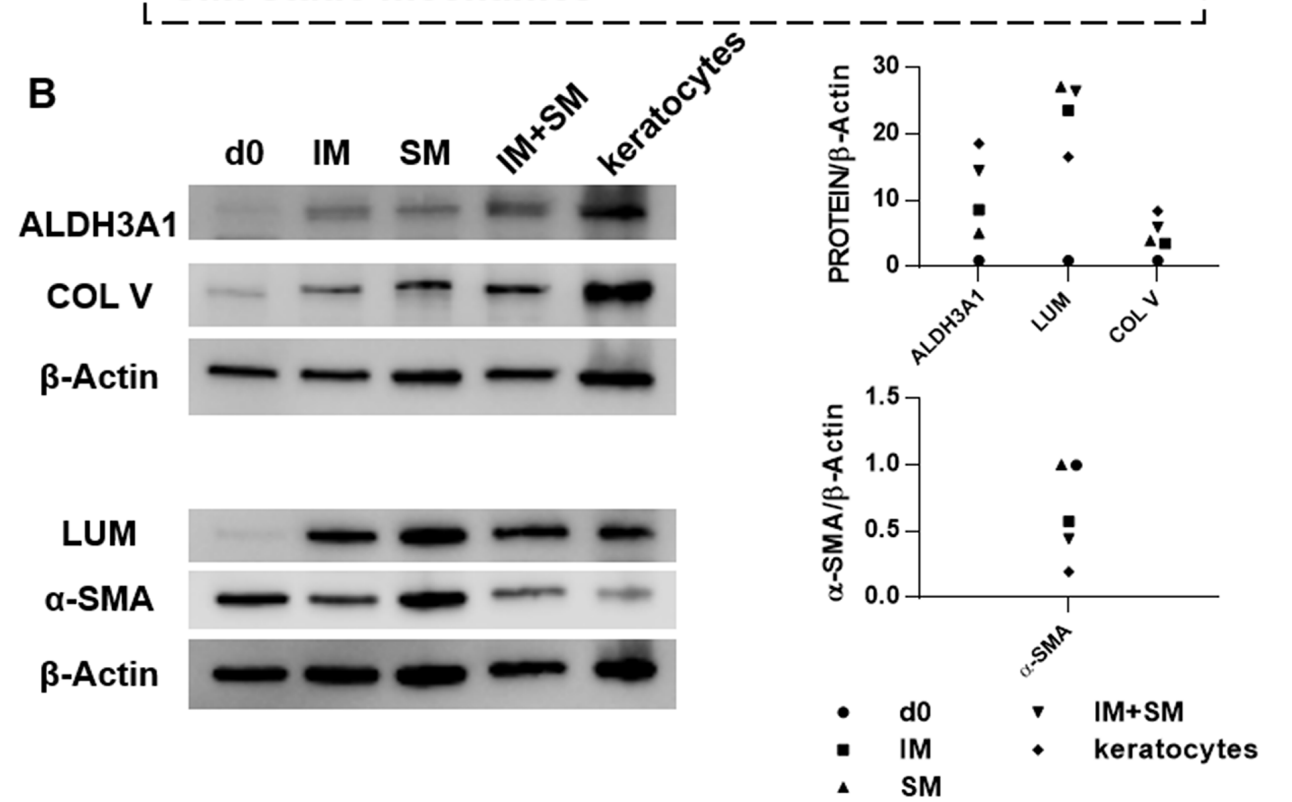

Figure 2 Mechanical stimulation and inducing medium (IM) synergistically promote keratocyte differentiation. PDLSCs were seeded on Bioflex 6-well plates, with treatment of IM, static mechanics (SM) or a combination of both (IM+SM) for 6 days. (A) Gene expression was evaluated by quantitative PCR. Levels at day 0 (unstimulated PDLSCS) were set as 1. The expression was compared between IM and SM, IM and IM+SM, SM and $I M+S M, I M+S M$ and primary in vitro cultured keratocytes at passage 2. "Significant difference at $P<0.05$. ${ }^{* *}$ Significant difference at $P<0.001$. N.S., no significant difference $(P \geq 0.05)$. (B) Protein expression was evaluated by Western blot. Densitometry was performed, and the ratio of protein/ $\beta$-Actin was calculated. Levels at day 0 (d0, unstimulated PDLSCs) were set as 1. PDLSCs, periodontal ligament stem cells.

collected as basal levels. Cultured primary human limbal keratocytes (at passage 2) were used as positive controls.

IM group: cells were cultured in the IM for 6 days.

SM group: cells were cultured in advanced DMEM plus $10 \%$ FBS, receiving 3\% static strain for 6 days.

IM+SM group: cells were cultured in the IM, receiving 3\% static strain for 6 days.
Refined time windows for the synergistic effect of mechanical strain and IM

PDLSCs $\left(2 \times 10^{5} /\right.$ well $)$ were seeded on Bioflex 6-well plate and cultured in regular growing medium for 1 day before treatments. The medium was changed in all wells at the same time. Samples were collected after 6 days of treatment for qPCR and Western blot. 
A

ALDH3A1

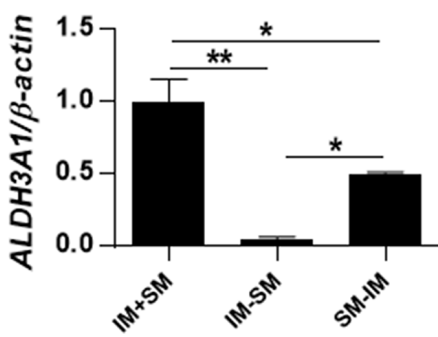

COL I

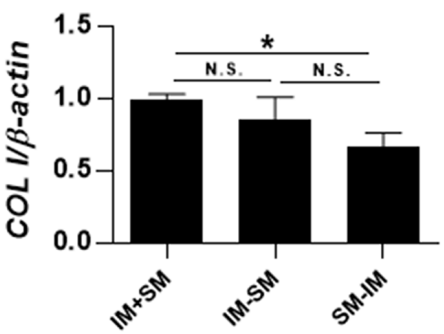

$C D 34$

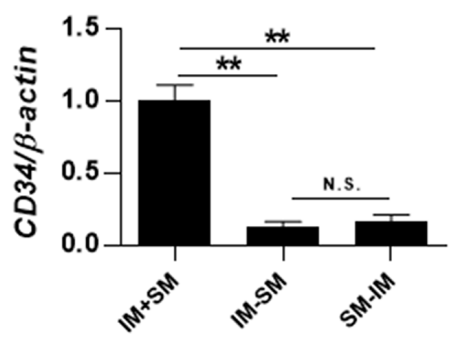

$\operatorname{COLV}$

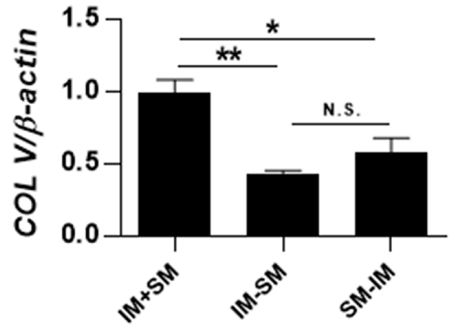

LUM

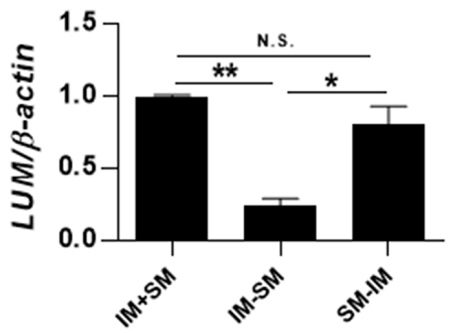

ACTA2

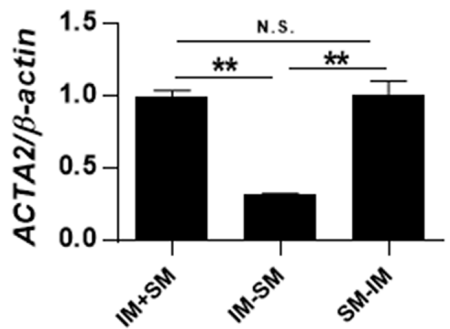

B

\section{IM+SM IM-SM SM-IM}
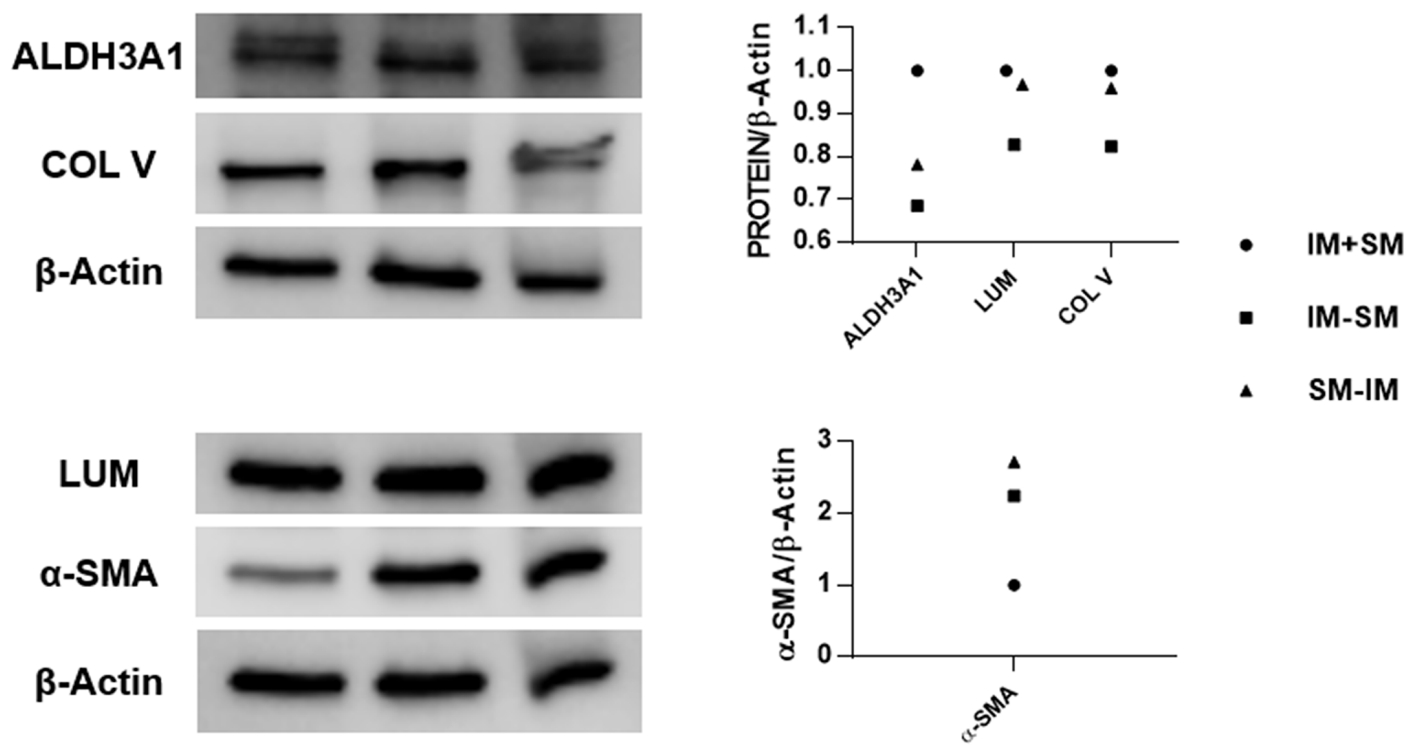

Figure 3 Mechanical stimulation and inducing medium (IM) exert superior effect on keratocyte differentiation when they are working together at the same time. PDLSCs were seeded on Bioflex 6-well plates, with treatment of IM and static mechanics (SM) together for 6 days (IM+SM), or IM for the first 3 days and SM for the following 3 days (IM-SM), or SM for the first 3 days and IM for the following 3 days (SM-IM). (A) Gene expression was evaluated by quantitative PCR. Levels of IM+SM were set as 1 . " Significant difference at $P<0.05$. ${ }^{* *}$ Significant difference at $P<0.001$. N.S., no significant difference $(P \geq 0.05)$. (B) Protein expression was evaluated by Western blot. Densitometry was performed, and the ratio of protein/ $\beta$-Actin was calculated. Levels of IM+SM were set as 1. PDLSCs, periodontal ligament stem cells.

IM+SM group: cells were cultured in the IM, receiving 3\% static strain continuously for 6 days.

IM-SM group: cells were cultured in the IM without strain for the first 3 days. After that, the cells were cultured in advanced DMEM plus 10\% FBS, receiving 3\% static strain for the following 3 days.

SM-IM group: cells were cultured in advanced DMEM plus $10 \%$ FBS, receiving 3\% static strain for the first 3 days. After that, the cells were cultured in the IM without strain for the following 3 days.

\section{Cell sheet formation}

PDLSCs $\left(4 \times 10^{5} /\right.$ well) were seeded on collagen membranes in Bioflex 6-well plates and cultured in growing medium. After 1 day, cells were cultured in the IM, receiving 3\% static strain 


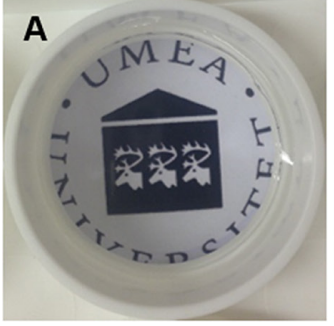

collagen membrane
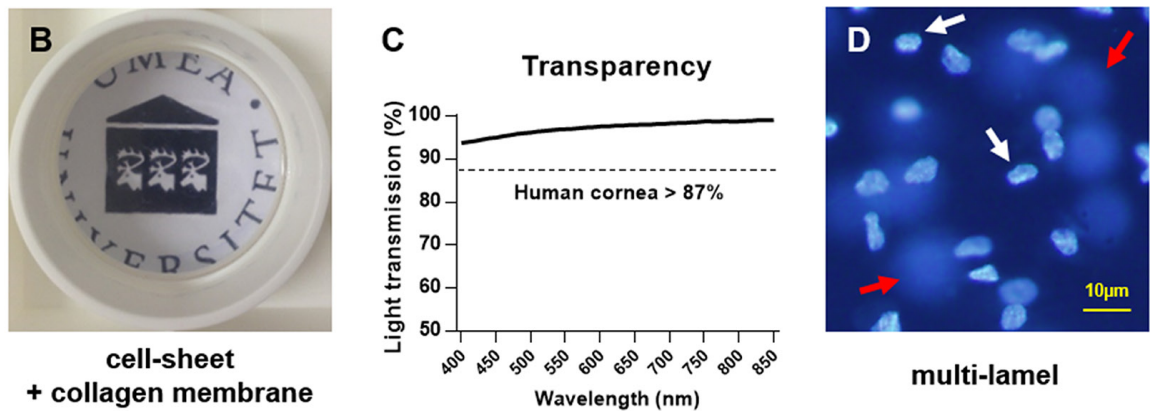

Figure 4 Mechanical stimulation and inducing medium generate a transparent and multilamellar cell sheet. PDLSCs were seeded on collagen I bonded Bioflex 6 -well plates and exposed to static mechanical strain and inducing medium for 12 days to form a cell sheet. The collagen membrane of the Bioflex 6-well plate itself (A) and the collagen membrane with the formed cell sheet (B) were both transparent. (C) The light transmission of the cell sheet was measured in the wavelength from $400 \mathrm{~nm}$ to $850 \mathrm{~nm}$. (D) The formed cell sheet was found to be a multilamellar structure, as revealed by DAPI staining; white arrows indicating nuclei that are focused on one layer, and red arrows indicating nuclei that were not focused and thus on another layer. PDLSCs, periodontal ligament stem cells.

continuously. Medium was changed every second day. After 12 days, the formation of a cell sheet was observed, and this cell sheet was collected for transparency measurement and immunofluorescence.

\section{RNA extraction and qPCR}

The same protocol was used for RNA extraction and cDNA reverse transcription as previously described. ${ }^{13}$ For qPCR performance, TaqMan Gene Expression Assay (Applied Biosystems, Carlsbad, California, USA) and SYBR Green reagents (Applied Biosystems) was used in this study. All probes and primers used here are summarised in online supplementary tables 1 and 2. Representative results of cells from two individuals are displayed as target gene expression normalised to housekeeping gene.

\section{Western blot analysis}

Cells from three replicate wells were pooled together as one sample. The detailed protocol for Western blot analysis is presented in online supplementary materials and methods. All antibodies used for Western blot are summarised in online supplementary table 3 .

\section{Transparency test}

Formed cell sheets with collagen membrane from the Bioflex 6-well plates were cut, cropped and placed in each well of a 12-well plate with PBS $(n=8)$. Unused collagen membrane immersed in PBS was set as control group $(n=8)$. Absorbance was determined from $400 \mathrm{~nm}$ to $850 \mathrm{~nm}$ in wavelength, with $5 \mathrm{~nm}$ intervals. The transparency was calculated using the formula: absorbance $=-\log (\%$ transmittance $/ 100)$.

\section{Immunofluorescence}

Cell sheets were collected and fixed in $4 \%(\mathrm{v} / \mathrm{v})$ paraformaldehyde. After permeabilisation with 1\% Triton X-100, the fixed cell sheets were blocked with 1:20 diluted normal serum. Samples were incubated with primary antibodies (see online supplementary table 3 ) overnight at $4^{\circ} \mathrm{C}$. Fluorescein-conjugated secondary antibodies (see online supplementary table 3 ) were incubated with samples after washing and finally addition of 4',6-diamidino-2-phenylindole (DAPI) to reveal the nuclei of the cells.

\section{Statistical analysis}

All gene expression data are shown as mean $\pm \mathrm{SD}$, and comparisons were carried out by performing one-way analysis of variance, with Bonferroni post hoc test for additional pair-wise comparisons between groups of interest. All experiments were performed in at least triplicate and were successfully repeated in PDLSCs derived from different patients. For all comparisons, $\mathrm{P}<0.05$ were considered statistically significant.

\section{RESULTS}

\section{Corneal-shaped mechanical stimulation promotes keratocyte differentiation}

To evaluate the effect of mechanical stimulation on the keratocyte differentiation, PDLSCs were seeded on a Bioflex 6-well plate, which was placed on a dome-shaped loading post in the Flexcell Tension System (figure 1A). After 7 days stimulation of static mechanics (SM d7), the expression of genes for keratocyte makers were significantly upregulated (figure $1 \mathrm{~B}$; ALDH3A1, 37.91-fold, $\mathrm{P}<0.001$; CD34, 2.69-fold, $\mathrm{P}<0.001$; LUM, 3.30-fold, $\mathrm{P}<0.05)$, as compared with the unstimulated PDLSCs, d0 ( $\mathrm{d} 0$ levels set as 1$)$. When compared with primary in vitro cultured keratocytes, the expression level of $A L D H 3 A 1$ in SM d7 group reached to $49 \%$ of that in keratocytes $(\mathrm{P}<0.001)$, no significant difference in CD34 $(\mathrm{P} \geq 0.05)$, and about threefold higher expression in LUM in SM d7 group $(\mathrm{P}<0.001)$. The expression of COL $I$ and $C O L V$ (main types of collagen in corneal stroma) and myofibroblast maker ACTA2 were also significantly increased at day 7 (SM d7) as compared with d0 (figure $1 \mathrm{~B} ; C O L I, 2.64$-fold, $\mathrm{P}<0.001$; COL V, 1.22-fold, $\mathrm{P}<0.05$; ACTA2, 1.58 -fold, $\mathrm{P}<0.001$ ), and both were significantly higher than that of primary in vitro cultured keratocytes. In contrast, the expression of genes for the tenocyte markers scleraxis $(S C X)$ and tenomodulin (TNMD) were significantly decreased both after 3 (SM d3; $\mathrm{P}<0.001)$ and 7 (SM d7; $\mathrm{P}<0.001)$ days of mechanical stimulation, as compared with $\mathrm{d} 0$ (figure $1 \mathrm{~B}$ ), which excludes a possible tenogenic differentiation by mechanical strain.

To elucidate the possible mechanism, the expression levels of integrin genes were evaluated (figure 1B). The expression of integrin alpha 2 (ITGA2) had a 2.46-fold increase at day 3 (SM $\mathrm{d} 3)$, as compared with $\mathrm{d} 0(\mathrm{P}<0.001)$. At $\mathrm{SM} \mathrm{d} 7$, all the four genes (ITGA1, ITGA2, ITGB1 and MYH10) were significantly upregulated as compared with $\mathrm{d} 0$, indicating that integrins may 


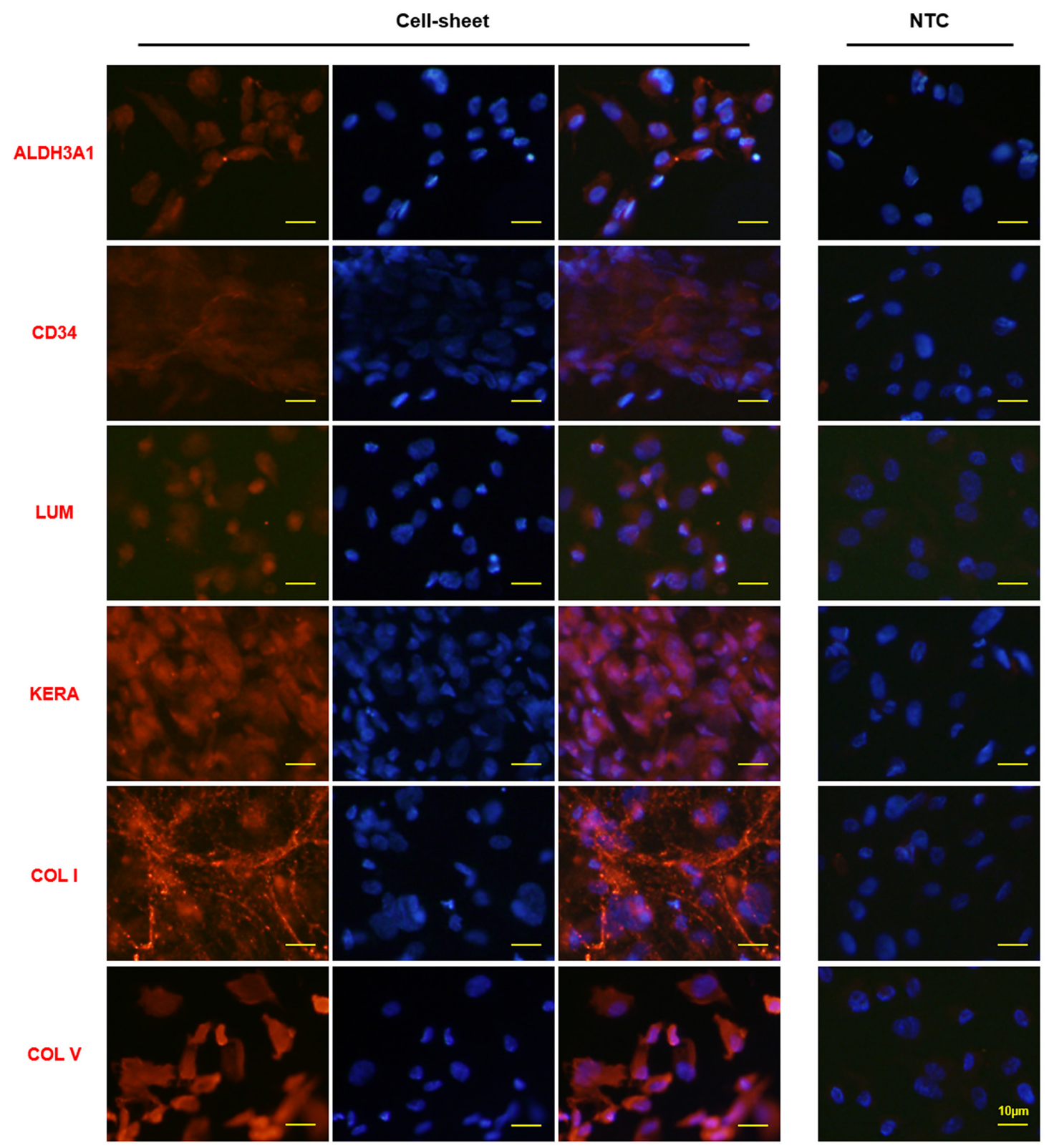

Figure 5 Typical markers of corneal stroma are expressed in the cell sheets constructed by PDLSCs exposed to mechanical stimulation and inducing medium. PDLSCs were seeded on Bioflex 6-well plates, with treatment of static mechanical strain and inducing medium for 12 days to form a cell sheet. The expression of typical corneal stroma markers was evaluated by immunofluorescence staining. The right column is the merged picture of left column (corneal stroma markers staining) and middle column (DAPI staining). 'NTC' indicates negative control without primary antibody. PDLSCs, periodontal ligament stem cells.

mediate mechano-transduction pathways during mechanically induced keratocyte differentiation.

\section{Mechanical stimulation and IM synergistically promote keratocyte differentiation}

To detect the possible synergistic effect between mechanical stimulation and IM on the keratocyte differentiation, gene expression was compared between groups subjected to IM alone, SM alone or a combination of IM and SM (IM+SM) (figure 2A) after treatment for 6 days. It was found that IM+SMgroup significantly increased the expression of keratocyte makers ALDH3A1 and LUM $(\mathrm{P}<0.05)$, as compared with both the IM and the SM group alone. Meanwhile, IM+SMgroup kept the same expression levels of COL $I$ as the other groups $(\mathrm{P} \geq 0.05)$.
The expression of CD34 and COL $V$ in the IM+SMgroup was lower than that in the IM group $(\mathrm{P}<0.001)$ but higher than that in the SM group $(\mathrm{P}<0.05)$. There was no significant difference on ACTA2 gene expression between the IM+SM and IM group $(\mathrm{P} \geq 0.05)$; however, there was significantly higher expression in the IM+SM group as compared with the SM group $(\mathrm{P}<0.001)$. When compared with primary in vitro cultured keratocytes at passage 2, the gene expression levels of keratocyte makers (ALDH3A1, CD34 and LUM) in IM+SMgroup were significantly higher $(\mathrm{P}<0.05)$, and the expression levels of the main types of collagen in corneal stroma (COL I and COL V) were comparable $(\mathrm{P} \geq 0.05)$. However, the expression level of myofibroblast marker ACTA2 was also significantly higher in the $\mathrm{IM}+\mathrm{SM}$ group, as compared with keratocytes $(\mathrm{P}<0.001)$. 
Protein expression of ALDH3A1, LUM, COL V and $\alpha$-smooth muscle actin ( $\alpha$-SMA) was evaluated by Western blot (figure $2 \mathrm{~B}$ ). It was noticed that IM+SMgroup had the strongest expression of ALDH3A1 and COL V and weakest expression of $\alpha$-SMA, as compared with the IM group, SM group and $\mathrm{d} 0$ group (unstimulated PDLSCs). The expression of LUM in the IM+SMgroup was slightly weaker than that in the SM group but stronger compared with that in IM group and $\mathrm{d} 0$ group. Nevertheless, it seems that the phenotype of the cells in the IM+SM group still had not reached the level of that for the in vitro cultured keratocytes, as the latter had even stronger expression of ALDH3A1 and COL $V$ and weaker expression of $\alpha$-SMA.

To refine the time window and optimal protocol for the synergistic effect of mechanical strain and IM, cells were treated with IM and SM together for 6 days $(\mathrm{IM}+\mathrm{SM})$, or IM for the first 3 days and SM for the following 3 days (IM-SM), or SM for the first 3 days and IM for the following 3 days (SM-IM). The results of qPCR showed that IM+SMgroup had the highest gene expression of keratocyte makers, collagens and myofibroblast maker, as compared with both the IM-SM and the SM-IM groups (figure 3A). The expression of ALDH3A1, LUM and COL V was evaluated on protein level, which confirmed the strongest expression in the IM+SMgroup (figure 3B). Interestingly, the weakest expression of the myofibroblast marker $\alpha$-SMA was found in the IM+SMgroup as compared with the other two groups, which differed from the gene expression result.

\section{Mechanical stimulation and IM generate corneal stroma-like cell sheets}

To explore the possible clinical application of PDLSCs for keratocyte differentiation, PDLSCs were treated for 12 days with a combination of mechanical stimulation and IM (IM+SM) to form a cell sheet on the collagen membrane. This cell sheet was transparent, as was the collagen membrane itself (figure 4A,B). The average light transmission of cell sheets $(n=8)$ were over $90 \%$ between the wavelengths of $400 \mathrm{~nm}$ and $850 \mathrm{~nm}$, which is higher than that of the native human cornea, that is, $>87 \%{ }^{14}$ (figure 4C). Furthermore, the cell sheet was found to be multilamellar in its structure, as shown with DAPI staining for the nuclei of the cells (figure 4D). The protein expression of typical markers of corneal stroma was evaluated by immunofluorescence (figure 5). Expression of ALDH3A1, CD34 and LUM was observed. High expression of KERA, the important proteoglycan of normal corneal stroma, was found in the cell sheet. Furthermore, the main collagen types in normal corneal stroma (COL I and COL V) were highly expressed.

\section{DISCUSSION}

This study explored the role of mechanical stimulation on capability of PDLSCs to differentiate towards keratocyte lineage and the synergistic effects of mechanical strain and keratocyte IM. Different inducing media have been adopted by researchers to differentiate stem cells towards keratocytes. However, some of the differentiation media require a long-term induction, such as 9 weeks, ${ }^{6}$ and some need to differentiate stem cells in a pellet model, ${ }^{15}$ which hampers the future translation to clinical use. Besides differentiation medium, coculturing stem cells with keratocytes is also a way to promote keratocyte differentiation ${ }^{5}$; this method essentially being a chemical induction as well. The effect of physical signals, such as mechanical stimulation, has not been reported on keratocyte differentiation yet. Our current study is the first, to our knowledge, to find that static mechanical strain promotes the differentiation of PDLSCs into keratocytes.

The combination of physical and chemical factors to induce cell differentiation is known in stem cells research. ${ }^{8}$ Tendon is quite similar to corneal stroma in structure, component and gene expression profile. ${ }^{16}$ Petrigliano and collaborators evaluated the differentiation effect of bone marrow stromal cells (BMSCs) towards teno-lineage, by using bFGF and uniaxial strain in a three-dimensional environment. ${ }^{17}$ The highest upregulation of tendon-related genes were found in the cells which were subjected to both mechanical stimulation and bFGF treatment for 21 days. ${ }^{17}$ Our current study combined static mechanical strain and chemical factors to induce the differentiation of PDLSCs into keratocytes. A synergistic effect on the expression of keratocyte makers was found when cells were cultured in IM and received 3\% dome-shaped static mechanical stimulation simultaneously. This is reasonable, as cells in vivo receive different kinds of stimulation simultaneously in their microenvironment, which warrants a systematic regulation during in vitro induced cell differentiation.

When different inducing factors are used together, a stepwise differentiation protocol is usually needed to achieve and optimise the desired effect. ${ }^{8}{ }^{18}$ Chen and collaborators overexpressed the tenocyte transcription factor SCX in human embryonic stem cell (ESC)-derived mesenchymal stem cells (MSCs) to drive the differentiation to tendon stem/progenitor cells. ${ }^{18}$ Subsequently, mechanical stress was introduced to achieve tenocyte commitment. ${ }^{18}$ In our current attempt, we also explored the possible effect of stepwise differentiation by setting time windows. However, no improvement was found in the stepwise differentiation group, which indicates that both mechanical stimulation and IM were needed together for the most efficient keratocyte differentiation in the current context. Promotion could possibly be made in future studies by refining the way of mechanical stress, finding more effective chemical factors and/or elaborating the way of combination.

Cell sheet strategy has been used in clinical applications, for example, oesophagus ${ }^{19}$ and cartilage. ${ }^{20}$ Regarding the cornea, cell sheet of autologous mucosal epithelial cells has been used for the repair of corneal epithelium in patients. ${ }^{21}$ Proulx and coworkers produced a complete human cornea in vitro by seeding endothelial and epithelial cells on each side of the keratocyte cell sheet, making it promising for corneal transplantation. ${ }^{22}$ Our present keratocyte-like cell sheet from differentiated PDLSCs was transparent, multilamellar and expressing markers of native human corneal stroma, making this model a highly interesting candidate for future clinical applications, after further in vitro and in vivo evaluation of cell-based therapies for cornea replacement/regeneration. One limitation of our current study is the lack of functional evaluation in vivo of the keratocyte-like cell sheet, such as cell survival, maintaining of phenotype, integration with the host corneal stroma tissue and possible immune response. These crucial issues need to be addressed in our future work.

Recently, Basu et $a l^{23}$ developed a therapy for corneal scarring by using autologous human limbal biopsy-derived stromal cells, which could be obtained with clinically established procedures and expanded under xeno-free autologous condition. Their work show great potential for autologous stem cell-based treatment of corneal stromal disease. However, the complicated components of culture medium and possible technical difficulty of stem cell isolation due to their sensitive location could hamper the future application of these cells to some extent. Highly proliferative PDLSCs could be cultured in vitro in an easy way (with basic medium supplemented with serum). They are accessible because dental tissues could be easily obtained from extracted teeth. In addition, they have the 
same immunomodulatory properties as BMSCs, ${ }^{24} 25$ and the immune-privileged characteristics of the human eye itself paves the way for allogenic PDLSCs transplantation. When compared with other alternative stem cells from non-corneal tissues, PDLSCs are similar to keratocytes in developmental origin and proteoglycan expression. ${ }^{7}$ Besides, tissues of ligaments/tendons have high similarity with corneal stroma, with aligned dense COL I, similar gene expression profile, especially for ECM collagens (collagen types I, III, V and VI) and proteoglycan (lumican, decorin and biglycan), ${ }^{16}$ submission to mechanical stress physiologically and similar pathological reactions. In conclusion, the characteristics of PDLSCs make them beneficial for medical corneal stroma applications.

In summary, the current study found a promoting effect of static mechanical stimulation on the differentiation of PDLSCs to keratocytes and that the effect was synergistic with the effect of IM. Cell sheets were constructed by the combined treatment of mechanics and chemicals, and these cell sheets resembled native human corneal stroma with expression of typical markers, which makes this technique a promising option in future clinical applications.

Acknowledgements The authors would like to thank Dr Marta Słoniecka and $\mathrm{Mr}$ Roine El-Habta for scientific advice.

Contributors JC and PD conceived the idea and designed the experiments. JC and WZ performed the experiments. All authors analysed the data and wrote the manuscript. PD and PK financially supported the study.

Funding Financial support was obtained by PD from the national Swedish Research Council (grant no: 521-2013-2612), the Swedish Society of Medicine, the Cronqvist foundation, the foundation Kronprinsessan Margaretas Arbetsnämnd för synskadade (KMA), the foundation Ögonfonden and Västerbotten County Council (VLL 'Spjutspetsmedel'). Financial support was furthermore provided to PD through a regional agreement (ALF) between Umeå University and Västerbotten County Council. In addition, this project was further financed by grants obtained by PK from Västerbotten County Council (VLL and TUA) and the Swedish Dental Society.

Competing interests None declared.

Patient consent Detail has been removed from this case description/these case descriptions to ensure anonymity. The editors and reviewers have seen the detailed information available and are satisfied that the information backs up the case the authors are making.

Ethics approval The study protocol was approved by the Regional Ethical Review Board in Umeå (2013-276-31M).

Provenance and peer review Not commissioned; externally peer reviewed.

Open Access This is an Open Access article distributed in accordance with the Creative Commons Attribution Non Commercial (CC BY-NC 4.0) license, which permits others to distribute, remix, adapt, build upon this work non-commercially, and license their derivative works on different terms, provided the original work is properly cited and the use is non-commercial. See: http://creativecommons.org/ licenses/by-nc/4.0/

(C) Article author(s) (or their employer(s) unless otherwise stated in the text of the article) 2018. All rights reserved. No commercial use is permitted unless otherwise expressly granted.

\section{REFERENCES}

1 Kumar P, Pandit A, Zeugolis DI. Progress in corneal stromal repair: from tissue grafts and biomaterials to modular supramolecular tissue-like assemblies. Adv Mater 2016:28:5381-99.
2 Sloniecka M, Backman LJ, Danielson P. Antiapoptotic effect of acetylcholine in fas-induced apoptosis in human keratocytes. Invest Ophthalmol Vis Sci 2016;57:5892-902.

3 Musselmann K, Alexandrou B, Kane B, et al. Maintenance of the keratocyte phenotype during cell proliferation stimulated by insulin. J Biol Chem 2005;280:32634-9.

4 Etheredge L, Kane BP, Valkov N, et al. Enhanced cell accumulation and collagen processing by keratocytes cultured under agarose and in media containing IGF-I, TGF- $\beta$ or PDGF. Matrix Biol 2010;29:519-24.

5 Zhang S, Espandar L, Imhof KM, et al. Differentiation of human adipose-derived stem cells along the keratocyte lineage in vitro. J Clin Exp Ophthalmol 2013;4.

6 Wu J, Du Y, Mann MM, et al. Bioengineering organized, multilamellar human corneal stromal tissue by growth factor supplementation on highly aligned synthetic substrates. Tissue Eng Part A 2013;19:2063-75.

7 Yam GH, Peh GS, Singhal S, et al. Dental stem cells: a future asset of ocular cell therapy. Expert Rev Mol Med 2015;17:e20.

8 Chen JL, Zhang W, Liu ZY, et al. Physical regulation of stem cells differentiation into teno-lineage: current strategies and future direction. Cell Tissue Res 2015;360:195-207.

9 Ogawa R, Orgill DP, Murphy GF, et al. Hydrostatic pressure-driven three-dimensional cartilage induction using human adipose-derived stem cells and collagen gels. Tissue Eng Part A 2015;21:257-66.

10 Zhang W, Chen J, Backman LJ, et al. Surface topography and mechanical strain promote keratocyte phenotype and extracellular matrix formation in a biomimetic 3D corneal model. Adv Healthc Mater 2017:6:1601238.

11 Chen J, Zhang W, Kelk P, et al. Substance P and patterned silk biomaterial stimulate periodontal ligament stem cells to form corneal stroma in a bioengineered threedimensional model. Stem Cell Res Ther 2017:8:260.

12 Słoniecka M, Le Roux S, Boman P, et al. Expression profiles of neuropeptides, neurotransmitters, and their receptors in human keratocytes in vitro and in situ. PLOS One 2015;10:e0134157.

13 Chen J, Chen P, Backman LJ, et al. Ciliary neurotrophic factor promotes the migration of corneal epithelial stem/progenitor cells by up-regulation of MMPs through the phosphorylation of akt. Sci Rep 2016;6:25870.

14 Fagerholm P, Lagali NS, Ong JA, et al. Stable corneal regeneration four years after implantation of a cell-free recombinant human collagen scaffold. Biomaterials 2014;35:2420-7.

15 Syed-Picard FN, Du Y, Lathrop KL, et al. Dental pulp stem cells: a new cellular resource for corneal stromal regeneration. Stem Cells Transl Med 2015;4:276-85.

16 Wu F, Lee S, Schumacher M, et al. Differential gene expression patterns of the developing and adult mouse cornea compared to the lens and tendon. Exp Eye Res 2008;87:214-25

17 Petrigliano FA, English CS, Barba D, et al. The effects of local bFGF release and uniaxial strain on cellular adaptation and gene expression in a $3 \mathrm{D}$ environment: implications for ligament tissue engineering. Tissue Eng 2007;13:2721-31.

18 Chen $\mathrm{X}$, Yin Z, Chen JL, et al. Force and scleraxis synergistically promote the commitment of human ES cells derived MSCs to tenocytes. Sci Rep 2012;2:977.

19 Ohki T, Yamato M, Ota M, et al. Prevention of esophageal stricture after endoscopic submucosal dissection using tissue-engineered cell sheets. Gastroenterology 2012;143:582-8

20 Sato $\mathrm{M}$, Yamato $\mathrm{M}$, Hamahashi $\mathrm{K}$, et al. Articular cartilage regeneration using cell sheet technology. Anat Rec 2014;297:36-43.

21 Nishida K, Yamato M, Hayashida Y, et al. Corneal reconstruction with tissueengineered cell sheets composed of autologous oral mucosal epithelium. $N$ Eng/ J Med 2004;351:1187-96.

22 Proulx S, d'Arc Uwamaliya J, Carrier P, et al. Reconstruction of a human cornea by the self-assembly approach of tissue engineering using the three native cell types. Mol Vis 2010:16:2192-201.

23 Basu S, Hertsenberg AJ, Funderburgh ML, et al. Human limbal biopsy-derived stromal stem cells prevent corneal scarring. Sci Trans/ Med 2014;6:266ra172.

24 Wada N, Menicanin D, Shi S, et al. Immunomodulatory properties of human periodontal ligament stem cells. J Cell Physiol 2009;219:667-76.

$25 \mathrm{Kim} \mathrm{HS}$, Kim KH, Kim SH, et al. Immunomodulatory effect of canine periodontal ligament stem cells on allogenic and xenogenic peripheral blood mononuclear cells. J Periodontal Implant Sci 2010;40:265-70. 